\title{
LATTICE COMPLETION OF THE METRIZABLE TOPOLOGIES
}

\author{
C. V. RIECKE \\ (Received 15 November 1972; revised 16 March 1973) \\ Communicated by E. Strzelecki
}

\section{Introduction}

Several classes of topological spaces which are either the infimum or supremum of a family of metrizable topologies in the lattice of topologies were given by Anderson [1]. In this paper we investigate the completion of the set of metrizable topologies in four distinct convergence structure lattices on an infinite set. We also observe the influence on these completions of the question regarding the existence of nonnormal ultrafilters which Čech [3] asserted could be neither proved nor disproved using present mathematical axiom systems.

We use the notation and terminology of [4], [5], and [6]. The reader may refer to one of these sources for the basic definitions with the exception that filters with empty intersection will be called free filters. We will denote by $C(S)$ (respectively $P_{s}(S), P(S), T(S)$ ) the complete lattice of all convergence structures (resp. pseudo-topologies, pretopologies, topologies) on a set $S$. The orderings in $P_{s}(S)$, $P(S)$ and $T(S)$ are the usual orderings induced by the ordering in $C(S)$.

An ultrafilter is termed normal by Čech [3] if it contains a countable subcollection with empty intersection. We will refer to any filter having this property as being normal. If $\mathscr{F}$ is any filter on $S$ and $x$ is in $S,(x, \mathscr{F})$ will signify the topology on $S$ discrete at all points $y$ other than $x$ and whose neighborhood filter at $x$ is $\mathscr{F} \cap \dot{x}$ where $\dot{x}$ is the ultrafilter generated by $\{x\}$.

\section{Lattice completion of the metric topologies}

Throughout $S$ will denote an infinite set as the proofs of the main theorems for finite sets reduce to trivial cases.

DEFINITION 1. A $T_{1}$ convergence structure $q$ on $S$ is primary if for each $x$ in $S$ and each free filter $\mathscr{F}$ which $q$-converges to $x, \mathscr{F}$ is normal.

DEFINITION 2. A $T_{1}$ pseudo-topology $q$ on $S$ is fundamental if for each $x$ in $S$ and each free ultrafilter $\mathscr{F}$ which q-converges to $x, \mathscr{F}$ is normal.

We may note that even if nonnormal ultrafilters fail to exist, a fundamental 
pseudo-topology is not necessarily a primary convergence structure as evidenced by the cofinite topology on an uncountable set.

The proof of the following proposition is immediate from the definitions and will be omitted.

Proposition 1. i) The set of all primary convergence structures on $S$ is a complete sublattice of $C(S)$.

ii) The set of all fundamental pseudo-topologies on $S$ is a complete, coatomic sublattice of $P_{s}(S)$.

In [7], Kent proved that a topology of the form $(x, \mathscr{F})$ is pseudo-metrizable if and only if $\mathscr{F}$ has a countable filter base. Since every pseudo-metrizable topology is metrizable if and only if it is $T_{1}$ we find that a $T_{1}$ topology of the form $(x, \mathscr{F})$ is metrizable if and only if $\mathscr{F}$ has a countable filter base.

THEOREM 1. The primary convergence structures (fundamental pseudotopologies) are the completion in $C(S)\left(\right.$ in $\left.P_{s}(S)\right)$ of the family of all metrizable topologies on $S$.

Proof. It is apparent that every metrizable topology on $S$ is both a primary convergence structure and a fundamental pseudo-topology.

If $q$ is any nondiscrete primary convergence structure on $S$, for each $x$ in $S$ and each normal filter $\mathscr{F}$ which $q$-converges to $x$, there exists a family of distinct free filters $\left\{\mathscr{G}_{\alpha} \mid \alpha \in I(x, \mathscr{F})\right\}$ each with countable filterbase whose filtersum is $\mathscr{F}$. Each $\left(x, \mathscr{G}_{\alpha}\right)$ is metrizable and $q=\inf _{C(S)}\left\{\sup _{C(S)}\left\{\left(x, \mathscr{G}_{\alpha}\right) \mid \alpha \in I(x, \mathscr{F})\right\} \mid x \in X\right.$, $x \in q(\mathscr{F}), \mathscr{F}$ normal $\}$.

For the corresponding result in $P_{s}(S)$, we observe that coatoms in the lattice of fundamental pseudo-topologies are those topologies of the form $(x, \mathscr{F})$ where $x$ is in $S$ and $\mathscr{F}$ is a normal ultrafilter on $S$. Then if $\left\{\mathscr{G}_{\alpha} \mid \alpha \in I(x, \mathscr{F})\right\}$ is a family of distinct free filters each with countable filterbase whose filtersum is $\mathscr{F}$,

$$
(x, \mathscr{F})=\sup _{P_{s}(S)}\left\{\left(x, \mathscr{G}_{\alpha}\right) \mid \alpha \in I(x, \mathscr{F})\right\} .
$$

The proof then follows from Proposition 1.

A consequence of Theorem 1 is that if nonnormal ultrafilters do not exist, the completion in $P_{s}(S)$ of the family of metrizable topologies is the lattice of $T_{1}$ pseudo-topologies. A quick examination of the cofinite topology on an uncountable set shows the corresponding result fails in $C(S)$ regardless of the existence of noonormal ultrafilters. A more comfortable situation exists in the lattices $P(S)$ and $T(S)$ where, with the aid of the next proposition, we observe that the existence of nonnormal ultrafilters does not prevent us from definitely establishing the completion of the family of metrizable topologies.

Proposition 2. For any free ultrafilter $\mathscr{F}$ on $S$, there exists a family of filters $\left\{\mathscr{G}_{\alpha}\right\}$ whose filtersum is $\mathscr{F}$ such that each $\mathscr{G}_{\alpha}$ is the intersection of a family of normal ultrafilters. 
Proof. For each $A$ in $\mathscr{F}$, let $\{\mathscr{H}(A, \beta) \mid \beta \in I(A)\}$ be the family of all normal ultrafilters containing $A$. Then $\mathscr{F}$ is the filtersum of $\{\mathscr{G}(A) \mid A \in \mathscr{F}\}$ where each $\mathscr{G}(A)$ is the intersection of $\{\mathscr{H}(A, \beta) \mid \beta \in I(A)\}$.

THEOREM 2. The $T_{1}$ topologies ( $T_{1}$ pretopologies) are the completion in $T(S)($ in $P(S))$ of the set of all metrizable topologies on $S$.

Proof. If $(x, \mathscr{F})$ is any $T_{1}$ topology on $S$ with $\mathscr{F}$ an ultrafilter, Proposition 2 implies $\mathscr{F}$ is the filtersum of a family of filters $\left\{\mathscr{G}_{\alpha}\right\}$ such that each $\mathscr{G}_{\alpha}$ is the intersection of a family of normal ultrafilters $\{\mathscr{H}(\alpha, \beta)\}$. Each filter $\mathscr{H}(\alpha, \beta)$ is the filtersum of a family of distinct free filters $\mathscr{K}(\alpha, \beta, \gamma)$ each with countable filterbase. Each $(x, \mathscr{K}(\alpha, \beta, \gamma))$ is metrizable and $(x, \mathscr{F})=\sup _{\alpha} \inf _{\beta} \sup _{y} \mathrm{~T}(S)(x, \mathscr{K}(\alpha, \beta, \gamma))$. Since each coatom in the coatomic sublattice of $T_{1}$ topologies belongs to the completion of the metrizable topologies, the proof for $T(S)$ follows immediately.

For the analogous result in $P(S)$, it is sufficient to observe that $P(S)$ is coatomic, the coatoms in $P(S)$ are precisely the coatoms of $T(S)$ and that if $(x, \mathscr{F})$ is a coatom in $P(S)$,

$$
(x, \mathscr{F})=\sup _{\alpha} \inf _{\beta} \sup _{\gamma(S)}(x, \mathscr{K}(\alpha, \beta, \gamma)) .
$$

\section{References}

[1] B. A. Anderson, Topologies comparable to metric topologies, (Proceedings of the Arizona State University Conference on General Topology, Tempe, 1967).

[2] A. M. Carstens, 'The Lattice of pretopologies on an infinite set', Pac, J. Math. 29 (1969), 67-71.

[3] E. Cech, Topological spaces, (Interscience, New York, 1966).

[4] B. V. Hearsey and D. C. Kent, 'Convergence structures', Port. Math. (1972), 105-118.

[5] D. C. Kent, 'Convergence function 5 and their related topologies', Fund. Math. 54 (1964) 125-133.

[6] D. C. Kent, 'Convergence quotient maps', Fund. Math. 65 (1969), 197-205.

[7] D. C. Kent, 'Decisive convergence spaces, Fréchet spaces and sequential spaces', Rocky Mountain J. Math 1 (1971), 367-374.

Cameron College

Lawton, Oklahoma, 73501

U.S.A. 